(Aus der Anatomie des Friedrichstädter Krankenhauses zu Dresden. Director: Geh. Med.-Rath Prof. Dr. Schmorl.)

\title{
Ueber Placentartuberculose.
}

\author{
Von \\ Dr. Hans Sehlimpert, \\ Secundärarzt, z. Z. Assistent an der Universitäts-Frauenklinik in Freiburg i. Br.
}

Wenn wir die bis jetzt vorliegende Literatur über Placentartuberculose durchsehen, finden wir 2 Arten von Arbeiten: 1. rein casuistische Mittheilungen - dies ist der grössere Theil der Arbeiten und 2. systematisch an einem grösseren Material angestellte Untersuchungen. Die Methoden der Untersuchung waren entweder der mikroskopische Nachweis der tuberculösen Veränderungen und der Tuberkelbacillen in der Placenta oder der Thierversuch an Meerschweinchen, die mit einzelnen Placentarstückchen oder Placentarbrei geimpft wurden.

$\mathrm{Zu}$ den zuletzt erwähnten Arbeiten, den systematischen Untersuchungen, gehören folgende: die von Schmorl und Geipel'1), die von $\mathrm{Bossi}^{2}$ ) und die jüngst erschienene Arbeit von Sitzenfrey $^{3}$ ) aus der Franquéschen Klinik. Der letztgenannten Arbeit ist ein ausführliches kritisch durchgearbeitetes Literaturverzeichniss beigegeben, auf das ich hier ausdrücklich verweisen möchte.

Die Mehrzahl der Autoren fanden in den von ihnen untersuchten Placenten bei schwerer Tuberculose und miliarer Tuberculose, aber auch bei beginnender Erkrankung der Mutter tuberculöse Veränderungen und Tuberkelbacillen, es liegen aber auch Beobachtungen, z. B. die von Bossi, vor, dem es mit Hülfe des Thierversuches nicht glückte, an 12 Placenten tuberculöser Mütter, No. XV.

1) Verhandlungen der Deutschen pathologischen Gesellschaft. 1904.

2) Archiv für Gynäkologie. 1906. No. 77. S. 21.

3) Die Lehre von der congenitalen Tuberculose. Berlin. 1909. S. Karger. 
die zur Einleitung der künstlichen Frühgeburt in die Klinik von Genua eingeliefert worden waren, Tuberkelbacillen nachzuweisen. Selbst wenn man die Beweiskraft steril ausgeführter Thierversuche zu diesem Zwecke zugiebt, so erschien uns doch nach unseren Erfahrungen dieses Ergebniss auffallend, und wir beschlossen ebenfalls systematische Untersuchungen in dieser Frage anzustellen. Wir wurden darin noch bestärkt, als eine Arbeit aus dem Institut für Hygiene und Bakteriologie der Universität Strassburg von Hamm und Schrumpf ${ }^{\mathbf{1}}$ ) erschien, die im Anschluss an die Beobachtung eines Falles, bei dem sie Tuberkelbacillen in der Placenta nachweisen konnten, aber keine im Fötus fanden, den auch von uns vertretenen Satz besonders hervorhoben, dass die intrauterine Uebertragung der Tuberculose kein häufiges Vorkommniss sei, die dann aber weiterhin auf die Verschiedenheiten der Resultate von Bossi und Schmorl hinwiesen und einige Sätze aufstellten, die - zum Theil sich widersprechend - geeignet sind, falsche Vorstellungen zu erwecken. Abgesehen davon, dass sich ihre Beobachtungen nur auf einen Fall erstrecken, fiel es auf, dass sie auf Grund der Untersuchungen von 200 Schnitten eine Placenta, in der Tuberkelbacillen mikroskopisch und experimentell sich nachweisen liessen, als frei von tuberculösen Veränderungen erklärten. Zunächst möchte ich hervorheben, dass auch der geübte Mikroskopiker im Anfang die kleinen Placentartuberkel sehr leicht übersehen kann. Jeder, der längere Zeit sich mit derartigen Untersuchungen beschäftigt hat, wird das bezeugen. Sodann halte ich Untersuchungen an 200 Schnitten für nicht hinreichend, zum mindesten, wenn ich dann aus dieser Einzelbeobachtung weitgehende Schlussfolgerungen ziehen will. Man braucht nicht, wie Schmorl es in einem seiner Fälle thut, gerade stets 2000 Schnitte zu untersuchen, sollte sich aber nicht scheuen, wenigstens 500 durchzumustern. Auch Sitzenfrey, der nach Schmorl und seinen Schülern ja die ausführlichsten Untersuchungen angestellt hat, weist darauf hin, wie oft nur langes Suchen zum Ziele führt und wie eine als negativ schon bei Seite gestellte Placenta bei frisch aufgenommener Untersuchung doch noch Tuberkel finden lässt. Sodann möchte ich folgenden Passus in den Ausführungen ron $\mathrm{Hamm}$ and Schrumpf festlegen ${ }^{2}$ ): "endlich möchten wir im Gegensatz zu

1) Centralbl. f. Bakteriologie. Bd. 43 . Originale.

2) 1. c. S. 311 . 
Schmorl auf die Nothwendigkeit von Impfversuchen mit dem tubercnloseverdächtigen Materiale hinweisen; denn bei spärlicher Anwesenheit von Tuberkelbacillen bietet die histologische Untersuchung nicht nur enorme Schwierigkeiten, sondern sie gewährt auch eine geringe Zuverlässigkeit. Hingegen dürfte es bei ausgiebig angestellten Thierversuchen sehr schwer sein, auch minimale Mengen von Tuberkelbacillen zu übersehen." Einige Zeilen später fahren sie fort: "Die Frage der Häufigkeit tuberculöser Veränderungen in der Placenta steht unseres Erachtens noch durchaus offen ... Der Nachweis von Tuberkelbacillen im Placentarsaft bei Fehlen jeglicher histologischer Veränderungen des Placentargewebes beweist selbstverständlich noch keineswegs eine Tuberculose der Placenta."

Die Autoren empfehlen also erst die Untersuchungen auf Tuberkelbacillen mit dem Thierexperiment, erklären aber dann von vornherein die dadurch gefundenen Resultate für nicht beweiskräftig. Da sie nun auch die histologische Untersuchung als schwierig und unzuverlässig ablehnen, bleibt es unklar, wie sie überhaupt den Nachweis der Placentartuberculose führen wollen.

Wenn ferner Hamm und Schrumpf darauf hinweisen, dass die Häufigkeit der von Schmorl und seinen Schülern gefundenen Placentartuberculose durch eine Besonderheit des Materials bedingt ist, so geben wir zu, dass es sich bei dem Schmorl'schen Material in der Mehrzahl der Fälle um schwere Phthisen der Mutter handelte. Es kann aber nicht zugegeben werden, dass die Bossi'sche Statistik gegen die Schmorl'sche ausgespielt wird, denn auch die Mütter, von denen die von Bossi untersuchten Placenten stammten, waren an schwerer Phthise erkrankt, die die Einleitung der künstlichen Frühgeburt nöthig machten. Gerade unter dem Material von Schmorl und Geipel aber finden sich positive Befunde bei mittelschwerer und auch bei beginnender Phthise (2 unter 9). Ich bin der Ansicht, dass Verschiedenheiten des Befundes durch Verschiedenheiten der Methoden des Nachweises bezw. durch Unterschiede in der auf die Untersuchung verwendeten Ausdauer erklärt werden. Es war daher mein Bestreben, eine Technik auszuarbeiten, die dem Ideal, die Placenta vollständig zu untersuchen, möglichst nahe kommt. Ich verfuhr folgendermaassen: Aus den verschiedensten Theilen der Placenta werden grosse Stücke (die resultirenden Schnitte waren meist ungefähr $5 \mathrm{~cm}$ lang und $3 \mathrm{~cm}$ breit) entnommen und in Paraffin eingebettet. Die Blöcke wurden serien- 
weise zerlegt und jeder 5.-10. Schnitt aufgefangen und auf $18 \mathrm{~cm}$ langen und $5 \mathrm{~cm}$ breiten Glasstreifen aufgezogen, die man sich leicht aus allep photographischen Platten schneiden kann. Eine solche Platte enthält ungefähr 10 Schnitte und wurde in entsprechenden Schalen gefärbt, entwässert u. s.w. Es erwies sich dies praktischer, als die sonst sehr empfehlenswerthe ${ }^{1}$ ) Photoxylinhautmethode, da bei dieser die Tuberkelbacillenfärbung nicht auszuführen ist. Es wurde stets nur die Färbung auf Tuberkelbacillen im Schnitt ${ }^{2}$ ) (Vorfärbung in Hämatoxylin, 1 Stunde Carbolfuchsin bei $37^{\circ}$ ) angewandt. Diese Färbung giebt so klare Bilder auch der histologischen Structur, dass eine besondere Färbung mit den gewöhnlichen Doppelfärbungen noch ausserdem (Ḧ̈matoxylin-Eosin) überflüssig ist. Die gefärbten und in Xylol aufgehellten Schnitte wurden principiell - um Zeit zu sparen - nicht in Canadabalsam eingeschlossen und mit Deckgläschen bedeckt, sondern im feuchten Zustand unter dem Mikroskop mit der Lupe (Zeiss $a_{3}$ ) durchsucht. Fanden sich dabei verdächtige Herde, so wurden diese Schnitte mit Deckgläschen versehen und dann mit den stärkeren Systemen und der Oelimmersion untersucht. Ich glaube, dass die angegebene Methode Kosten und Zeit in hohem Maasse spart und möchte sie ausser zu diesem Zwecke auch sonst empfehlen.

Das Material zu den systematischen Untersuchungen entstammte der gynäkologischen Abtheilung ${ }^{3}$ ) und in einem Falle der II. inneren Abtheilung ${ }^{4}$ ) des Friedrichstädter Krankenhauses. Das Material an geburtshülflichen Fällen ist dort sehr klein und so kam es, dass wir in der Zeit vom Februar bis October 1908 nur 7 Placenten erhielten von Müttern, bei denen Tubereulose in der Schwangerschaft constatirt war.

Ich füge dem noch kurz 4 rein casuistische Mittheilungen über 4 Fälle von Placentartuberculose hinzu, die hier in den letzten Jahren beobachtet wurden und noch nicht veröffentlicht sind, die alle das Gemeinschaftliche haben, dass sie von Frauen stammen, die während der Schwangerschaft unentbunden starben und zur Section kamen. 1909. S. 71.

1) Schmorl, Die pathologisch-histologisehen Untersuchungsmethoden.

2) Idem. S. 302.

3) Director: Geh. San.-Rath Dr. Osterloh.

4) Director: Prof. Dr. Rostoski. 


\section{Systematische Untersuchungen.}

\section{A. Positive Fälle.}

1. Bertha Helene E., Hausarbeitersehefrau, 29 Jahr.

Befindet sich wegen chronischer Lungentuberculose auf der Ersten inneren Abtheilung des Friedrichstädter Krankenhauses (Prof. Püssler) und wird von dort am 4.2.08 nach der gynäkologischen Station (Geh. San.-Rath Osterlob) verlegt, wo am 8. 2. der typische conservative Kaiserschnitt an der Sterbenden ausgeführt und ein lebendes Kind erzielt wird.

Sectionsprotocoll der Mutter vom 10.2. No. 106.

Meningitis tuberculosa basalis. Chronische lymphangitisehe Form der Lungentuberculose. Anthrakose beider Spitzen. Tuberculöse Pleuritis links. Vereinzelte miliare Tuberkel in $\mathrm{Mlilz}$ und Nieren. Verkäsung der linken Nebenniere. Tuberculöse Darmgeschwüre. Leichte Hypertrophie des rechten Herzventrikels. Oedem der Leber, Oedem und Anämie der Nieren. Sectio caesarea uteri puerperalis.

Kind: Mädehen, Frühgeburt von $1310 \mathrm{~g}$ Gewicht. Stirbt unmittelbar nach der Geburt.

Sectionsprotocoll des İindes vom 10. 2. No. 107.

Frühgeburt im 6. Monat. Hyperämie der Unterleibsorgane. Vereinzelte kleine Luftbläschen in des sonst atelektatischen Lungen.

Placenta: Es finden sich an 3 verschiedenen Stellen tuberculöse Gewebsveränderungen mit positivem Bacillenbefund. Alle 3 Tuberkel gehören zum Typus I nach Schmorl (intervillär gelegene). Einer ist über stecknadelkopfgross mit verkästem Centrum und zellreicher Peripherie (Lymphocyten, Leukocyten, Epitheloidzellen) und einzelnen Riesenzellen. Ausser einem in einer Riesenzelle gelegenen Tuberkelbacillus finden sich noch mässig zahlreiche zwischen den Zellen der Peripherie und im Centrum des Tuberkels. Mehrere kleine Zotten sind in den Tuberkel einbezogen. Ausserdem liegen noch 2 kleine, vorwiegend aus Rundzellen bestehende wenige Bacillen enthaltende Tuberkel zwischen einzelnen Zotten, in die sie ohne scharfe Grenze übergehen.

2. Magdalene F, Glasarbeitersehefrau, $42 \mathrm{Jahr}$.

Wird am 24. 4. 08 wegen schwerer Kehlkopf- und beiderseitiger Lungentuberculose ins Krankenhaus zur Einleitung der künstlichen Frühgeburt eingeliefert. Nach Einlegen eines Hetreurynters und Blasensprengung erfolgt am 26. 4. die spontane Geburt eines lebenden Knaben. Wochenbettsverlauf afebril. Patientin wird am 4. 5. ungeheilt auf ihren Wunsch entlassen. Nähere Angaben über ihr weiteres Befinden fehlen.

Kind: $1830 \mathrm{~g}$ schwerer Knabe. Stirbt 7 Stunden nach der Geburt an Lebensschwäche.

Sectionsprotocoll No. 325.1908.

Frühgeburt im 7. Monat. Lufthaltige Lungen, allgemeine Lebensschwäche. Keine Zeichen von Tuberculose.

Placenta: Nach längerem Suchen finden sich 2 kaum hirsekorngrosse Tuberkel vom Typhus der intervillären Tuberkel. Das Centrum ist bei beiden schon verkäst, in den Randpartien findet sich eine Anhäufung von Lymphocyten, Epitheloidzellen und Riesenzellen. Zahlreiche Tuberkelbacillen in den Riesenzellen und zwischen den Zellen der Randpartie gelegen. Die Zotten der Umgebung sind mit beiden Tuberkeh verklebt und durch Randzellinfiltration theilweise zerstört. 
3. Anna H., Arbeiterin, $20 \mathrm{Jabr}$.

Wird am 15.6.08 wegen Tuberculose der gesamten rechten Lunge und der linken Lungenspitze und Kehlkopftuberculose auf der Ersten inneren Abtheilung aufgenommen. Es besteht Gravidität im 8. Monat. Vom 28. 6. an hat sie ständig Temperatur bis $39,0^{\circ}$. Am 2. 8. treten schwache Wehen auf, sie wird deshalb auf die gynälologische $\mathrm{Ab}$ theilung verlegt. Hier am selben Tage Geburt eines lebensschwachen Mädchens im 8. Monat. Im Wochenbett hochfebrile Temperaturen bis 40,0 $0^{\circ}$ Zunehmender Kräfteverfall. Am 7. 8. Rückverlegung auf die Erste innere Abtheilung. Hier Exitus am 12. 8.

Sectionsprotocoll der Matter No. 600. 1908.

Chronische ulceröse Lungenphthise. Grosse in schiefrig indurirtes Gewebe eingebettete Caverne im rechten Oberlappen. Klẹine Cavernen im rechten Unterlappen. Aeltere und frische Aspirationsherde in den übrigen Lungentheilen. Tuberculöse Bronchitis. Pibrinöse tuberculöse Pleuritis. Schwere Kehlkopf- und Trachealtuberculose. Tuberculose der Halslymphdrüsen. Vereinzelte miliare Tuberkel in Milz, Leber und Nieren. Parenchymatöse Degeneration der Nieren. Kalkinfarcte in den Papillenspitzen. Tuberculöse Geschwüre im Cöcum und Dickdarm. Hyperämie und Oedem des Gehirns. Uterus puerperalis mit ausgedehnten retrabirten Placentarresten. Ausgedehnter Riss in der Portio.

Kind: Lebensschwaches Mädchen von $1550 \mathrm{~g}$ Gewicht. Icterus neonatorum. Exitus am 8. 8, an Lebensschwäche.

Sectionsprotocoll des Kindes No. 590. 1908.

Icterus neonatorum. Blutungen in die Meningen. Pneumonie in beiden Unterlappen. Harnsäureinfarcte.

Placenta: Nach Durchsuchen von ungefähr 20 Schnitten findet sich ein knapp erbsengrosser intervillärer Tuberkel, dessen Centrum verkäst ist und dessen Rand zablreiche Lymphocyten, Leukocyten, Epitheloidzellen und mehrere Langhans'sche Riesenzellen aufweist. In den Herd sind zahlreiche Zotten einbezogen und durch Infiltration zum Theil zerstört. Noch im Inneren des nekrotischen Herdes lassen sieh die Conturen der Zotten theilweise unscharf erkennen. Die in der Umgebung des Tuberkels gelegenen intervillären Räume sind mit einem homogenen Gerinnsel erfüllt. Tuberkelbacillen finden sich spärlich im nekrotischen Theil und in einigen Riesenzellen. Ausserdem zeigen noch 2 Stellen Gewebsveränderungen vom Typus der intervillären Tuberkel mit centraler Nekrose und Riesenzellen. Beide Herde sind ungefähr stecknadelkopfgross. Tuberkelbacillen liessen sich in ihnen nicht nachweisen.

4. P. B., Näherin, 23 Jahr.

Wird am 11. 9. 08 auf der Ersten inmeren Abtheilung wegen fortschreitender Lungentuberculose aufgenommen. Es bestebt keine Kehlkopftuberculose. Im Sputum enorme Mengen von Bacillen. Schwangerschaft im ১. Monat. Zunehmender Kräfteverfall und Ausbreitung des tuberculösen Processes über beide Lungen. Da Wehen einsetzen, wird sie am 9. 10. auf die gynäkologische Abtheilung verlegt und hier spontan von einem Knaben entbunden. Unmittelbar nach der Eutbindung starker Collaps. Hochfebriler Verlauf des Wochenbettes ohne entsprechenden Befund an den Genitalien. Fortschreitende Verschlechterung des Lungenbefundes. Am 13. 10. Rückverlegung auf die Erste innere Abtheilung und hier am 15. 10. Exitus.

Sectionsprotocoll der Mutter No. 735. 1908. 
Tuberculöse Lungenphthise. Kleine Cavernen in beiden Spitzen. Ausgedehnte käsige confluirende Pneumonien in beiden Lungen. Fibrinöse Pleuritis. Adhäsive Pleuritis über beiden Spitzen. Verfettung der Herzmusculatur. Kleine lenticuläre Geschwüre in der Trachea. Tuberculose der Bronchialdrüsen. Acuter Milztumor. Miliare Tuberkel in den Nieren. Nephritis. Miliare Tuberkel in der Leber. Kleine Nekrosen in der Leber. Staungsleber. Magenkatarrh. Tuberculöse Darmgeschwüre. Uterus puerperalis, in Rückbildung begriffen.

Kind: Knabe, schwächliche Frühgeburt von $1550 \mathrm{~g}$ Gewicht. Künstliche Ernährung und Wärmezufuhr. Am 12. 10. Exitus.

Sectionsprotocoll des Kindes No. 728. 1908.

Lebensschwäche, totale Atelektase der Lungen. Hyperämie der Unterleibsorgane.

Placenta: Bei der makroskopischen Untersuchung fällt sofort ein über stecknadelkopfgrosses an der fötalen Seite der Placenta gelegenes Knötchen auf. Ein von diesem angefertigtes Quetscbpräparat zeigt ausserordentlich zahlreiche Tuberkelbacillen. An den aus den verschiedensten Theilen der Placenta entnommenen Schnitten gelingt es trotz langen Suchens nicht, eine auf Tuberculose verdächtige Gewebsveränderung oder sonst Tuberkelbacillen nachzuweisen.

5. Ida K., Schneiderin, 24 Jahr.

Seit 14 Tagen bestehende Durchfälle, Auswurf und Husten veranlassen Patientin am 4. 8. die Erste innere Abtheilung des Krankenhauses aufzusuchen. Es besteht Tuberculose beider Lungenspitzen, rechts stärker als links, keine Kehlkopftuberculose. Schwangersehaft im 6 . bis 7. Monat. Am 22. 9. Verlegung nach der gynäkologischen Abtheilung zur Finleitung der künstlichen Frühgeburt. Nach mehrmaliger Dilatation mit dem Laminariastift und Wendung wird ein lebensschwaches Kind durch Extraction am Fuss entwickelt. Manuelle Placentarlösung. Hochfebriles Wochenbett. Temperatur sinkt nach Abgang von Placentarresten am 3. 10. zur Norm. Rückverlegung auf die Erste innere Abtheilung. Hier tritt am 24. 12. unter anhaltend hohem Fieber und Ausbreitung des tuberculösen Processes über beiden Lungen Exitus ein.

Sectionsprotocoll der Mutter No. 903. 1908.

Lungentuberculose. Kleine Cavernen in der rechten Spitze. Zahlreiche frischere und ältere Aspirationsberde über beiden Lungentheilen. Adhäsive Pleuritis beiderseits. Frische Embolie im linken Unterlappen. Geringe Hypertrophie des linken Ventrikels. Perisplenitis. Miliare Tuberkel der Milz, Leber und Nieren. Braune Atrophie der Leber. Tuberculose des Peritoneums. Käsige Endometritis und Salpingitis. Geringe Cystitis. Schwerer Nagenkatarrh. Geringe folliculäre Schwellung im Darm.

Kind: Lebensschwache Frühgeburt von $1550 \mathrm{~g}$ Gewicht. Mädchen. Icterus neonatorum. Exitus am 8. 8.

Sectionsprotocoll des Kindes No. 590. 1908.

Icterus neonatorum. Blutungen in die Meningen. Pueumonie in beiden Unterlappen. Harnsäureinfarcte.

Placenta: Nach langem Suchen fanden sich in der Decidua basalis 2 tuberkelbacillenhaltige Rundzelleninfiltrate. Es waren bei der Durchmusterung der Schnitte schon mehrmals in der Decidua Rundzelleninfiltrate aufgefallen. Es gelang aber nur in 2 von diesen TuberkeIbacillen nachzuweisen. Das Centrum der Herde bestand aus käsigen 
Massen mit einzelnen zum Theil staubförmigen mit Hämatoxylin sich stark färbenden Kerntrümmern. In der Peripherie fanden sich Lymphocyten und Leukocyten. Deciduazellen wurden erst in einiger Entfernung von den Herden geseben. Die Tuberkelbacillen lagen im käsigen $\mathrm{Ge}-$ webe nahe der Infiltrationszone und waren nicht sehr zablreich. Riesenzellen wurden in den Deciduaherden, worauf ja auch Schmorl schon hingewiesen hat, nicht gefunden.

6. Martha L., Wirthschafterin, 29 Jahr.

Leidet seit ungefähr 8 Wochen an Husten und Heiserkeit und wird wegen einsetzender Weben am 15. 10. aufgenommen (gynäkologische Abtheilung). Es besteht Schwangerschaft im 8. Monat. Die Untersuchung ergiebt beiderseitige fortschreitende Lungentuberculose und Kehlkopftuberculose. Am selben Tage erfolgt spontane Geburt eines lebensschwachen Mädchens. Am nächsten Tag Temperaturanstieg auf $40^{\circ}$. Am 17. 10. wird sie trotz des bestehenden Fiebers von $40^{\circ}$ gegen Unterzeichnung eines Berichts auf ihren Wunsch hin entlassen und soll zu Haus nach 2 Tagen gestorben sein. Section unterblieb.

Kind: $1820 \mathrm{~g}$ schweres Mädchen. Stirbt am 20. 10. an Lebensschwäche.

Sectionsprotocoll No. 750.1908.

Lufthaltige Lungen, keine Zeichen von Tuberculose. Allgemeine Schwäche.

Placenta: Auf der mütterlichen Seite in der Mitte der Placenta finden sich mehrere stecknadelkopfgrosse weissliche Knötchen. Ein Ausstrichpräparat, das von einem von diesen angefertigt wird, enthält mehrere Tuberkelbacillen. Schon nach Durchmusterung weniger Präparate findet sich ein intervillär gelegener ungefähr hirsekorngrosser ziemlich junger zellreicher Tuberkel, der zwei Zotten, deren Epithel arrodirt ist, anliegt. Verkäsung fehlt. Er besteht vorwiegend aus Lymphocyten, ausserdem sind einige Epitheloidzellen und Riesenzellen nachzuweisen. Zahlreiche Tuberkelbacillen liegen theils zwischen den Zellen, theils in den Riesenzellen eingeschlossen.

\section{B. Negative Fälle.}

7. Anna C., Schneidersebefrau, 30 Jahr.

Hat laut mündlicher Mittheilung im Juni wegen fortschreitender Lungentuberculose bei bestehender Schwangerschaft im 6. Monat die Zweite immere Abtheilung aufgesucht, wo Ende Juni die Frühgeburt einés unreifen todten Kindes im 6. Monat erfolgte.

Sectionsprotocoll der Mutter No. 568. 1908.

Ausgedehnte Lungentuberculose. Grosse Cavernen in beiden Oberlappen sowie Mittel- und rechtem Unterlappen. Aspirationstuberculose. Adhäsive Pleuritis beiderseits. Tuberculöse Bronchitis, Tracheitis, Laryngitis. Tuberculose der Bronchial- und Halslymphdrüsen. Magenkatarrh. Darmtuberculose und Tuberculose der Mesenterialdrüsen. Miliare Tuberkel in Leber, Milz und Nieren. Milztumor. Abort, in Rückbildung begriffener Uterus mit Placentarresten. Tuberculose der Vagina.

Das Kind wird uns in Formalin zusammen mit der Placenta überschickt. Die Zeichen der Reife fehlen, frühgeborener Knabe. An den inveren Organen keine Zeichen von Tuberculose. 


\section{Nicht systematisch untersuchte Fälle.}

Alle 4 Fälle stammen aus dem Friedrichstädter Krankenhaus. Es war uns nicht möglich Krankengeschichten zu erhalten. Von allen 4 Müttern, die sämmtlich unentbunden und in ziemlich frühen Stadien der Schwangerschaft starben, liegen die Sectionsprotocolle vor.

8. Sectionsprotocoll No. 689. 1903.

Anna Eruestine H., Lampenwärtersehefrau, 25 Jahr.

Aeltere ulceröse Tubereulose im linken Oberlappen. Frische in Zerfall begriffene Aspirationsherde in der rechten Spitze. Hyperämie der linken Lunge. Kleine vereinzelte Aspirationsherde in beiden UnterJappen. Tuberculose der bronchialen Lymphdrüsen. Diffuse Bronchitis. Anämie des Herzfleisches und der Nieren. Parenchymatöse Degeneration der Leber mit vereinzelten Tuberkeln. Magenkatarrh. Uterus gravidus 4. bis 5. Monat. Tuberculöse Geschwüre im Dickdarm. Vereinzelte Tuberkel in Milz und Nieren.

Placenta: Es finden sich ganz vereinzelte intervillöse Tuberkel und ein mässig ausgedehnter nekrotischer Herd in der Decidua. In den intervillösen Tuberkeln lassen sich keine Bacillen nachweisen. In dem nekrotischen Deciduaherd, der durchaus keine typische tuberculöse Structur anfweist, liegen zablreiche Tuberkelbacillen.

9. Sectionsprotocoll No. 353. 1904.

Ulceröse Lungenphthise. Adhäsive Pleuritis links. Ausgedehnte Cavernen im linken Oberlappen. Käsige Pneumonie im rechten Oberlappen. Aspiratiousherde in den übrigen Lungentheilen. Bronchitis und Tracheitis. Tuberculose der bronchialen Lymphdrüsen. Darmtuberculose. Parenchymatöse Degeneration beider Nieren. Miliare Tuberculose in Nieren, Milz und Leber. Milztumor. Gravidität im 5. Monat.

Placenta: Erst nach langem Suchen glückt $\mathrm{es}, 2$ tuberkelbacillenhaltige an der Oberfläche von Zotten gelegene Tuberkel mit Riesenzellen zu finden,

10. Sectionsprotocoll No. 772.1905.

Anna Else W., Fabrikarbeiterin, 19 Jahr.

Floride ulceröse Lungenphthise. Caverne im rechten Oberlappen. Pleuritis chronica adhaesiva. Hypertrophie des rechten Herzventrikels. Miliare Tuberkel in den Nieren. Tuberculöse Geschwüre im Kehlkopf, Cöcum und Colon. Verkreideter Cysticercus der Leber. Uterus gravidus.

Placenta: Auf der Schnittfläche durch die Placenta fallen mehrere im Gewebe gelegene grau-gelbliche Knötchen von Hirsekorngrösse auf, die herausgehoben und ausgeschnitten werden. Es finden sich in ihnen zahlreiche Tuberkelbacillen. Schnittpräparate von der Placenta wurden versebentlich nicht angefertigt.

11. Sectionsprotocoll No. 28. 1906.

Hulda Martha Sch., Strassenbabuwagenführersehefrau, 25 Jahr.

Hochgradige Lungentuberculose. Caverue im linken Oberlappen. Frische käsige Pneumonien in der übrigen Lunge, zum Theil in Erweichung begriffen, desgleichen im rechten Oberlappen, weniger ausgedehnt im rechten Unterlappen. Doppelseitige adhäsive Pleuritis. Hypertrophie des rechten Herzventrikels mit wandständigen Thromben und subendocardialer Verfettung. Kehlkopf- und Trachealtuberculose. 
Tuberculose der Halslymphdrüsen. Milztumor mit zablreichen Tuberkeln. Miliare Tuberkel in den Nieren. Magenkatarrh. Ausgedehnte Darmund Mesenterialdrüsentuberculose. Hyperämie der weichen Hirnhäute. Uterus gravidus im 4. Monat.

Vereinzelte kleine nekrotische bacillenhaltige Herde in der Decidua basalis, Blutungen der letzteren. Dicht oberhalb d. h. placentarwärts von diesen Herden spärliche Tuberkel, die zum Theil an Haftzotten sitzen.

Placenta: Es finden sich vereinzelte kleine nekrotische Herde in der Decidua basalis, in denen Tuberkelbacillen nachgewiesen werden können. Die Decidua ist von Blutungen stark durchsetzt.

Fasse ich epikritisch das Gefundene zusammen, so ergiebt sich Folgendes:

1. Bei systematisch angestellten Untersuchungen an Placenten tuberculöser Mütter wurden positive Befunde unter 7 Fällen 5 mal durch das histologisehe Präparat und den Bacillennachweis, 1 mal nur durch den Bacillennachweis im Ausstrich erhoben, also in 71,4 pCt. bezw. 85,7 pCt. der Fälle.

2. Die Casuistik über Placentartuberculose ist um 10 positive Fälle vermehrt (darunter 2 mit Bacillennachweis im Ausstrich).

Es ergeben sich also Resultate, die sich denen von Schmorl and Geipel, Sitzenfrey u. s. w. anschliessen und im Gegensatz zu denen von Bossi, Ascoli und Hamm und Schrumpf stehen.

Dem histologischen Bild nach handelte es sich 5 mal um die intervillöse Form der Placentartuberkel und 3 mal um Rundzelleninfiltrate in der Decidua. Diese letzt erwähnten 3 Placenten stammten aus relativ frühen Schwangersehaftsmonaten. Bei den 2 Placenten, bei denen Tuberkelbacillen im Ausstrich nachgewiesen wurden, besteht eine gewisse Wahrscheinlichkeit, dass es sich bei den so untersuchten verdächtigen Knötchen ebenfalls um intervilläre Tuberkel handelte. Alle untersuchten Placenten stammen von Müttern mit schwerer allgemeiner und fortgeschrittener Tuberculose. Durch die Beobachtung bei Fall 5 und 8-11 ist wiederum bestätigt, dass auch schon in den früheren Schwangerschaftsmonaten ein Einwandern der Bacillen in die Placenta stattfindet.

Zum Schluss möchte ich noch kurz meine Ansicht auf Grund der über Placentartuberculose vorliegenden Untersuchungen über die pathologische Bedeutung der Placentartuberculose und über die Möglichkeit des Uebertrittes von Tuberkelbacillen von der Placenta auf den Fötus auseinandersetzen:

Man kann die Placentartuberkel entweder als Reactionserscheinung des mütterlichen Organismus auf die Infection mit 
Tuberkelbacillen, als eine Abwehrvorrichtung auffassen oder aber man sieht sie als Etappen der Krankheit auf ihrem Wege von der Mutter zum Kinde an, als den Beginn der Metastasirung der mütterlichen Tuberculose im Sinne Lubarsch's.

Ich bin der Ansicht, dass die erste Anschaunng die richtigere ist. Ebenso wie sich bei Tuberculosen, bei denen Tuberkelbacillen in's Blut kommen, ausserordentlich häufig miliare Tuberkel in der Milz, Leber und den Nieren bilden, können bei bestehender Schwangerschaft auch miliare Tuberkel in der Placenta entstehen. Es braucht dabei nicht nur an die Fälle von allgemeiner Miliartuberculose gedacht zu werden, sondern es kämen hierfür alle Fälle, auch beginnende Tuberculose, in Betracht, bei denen ja, wie wir aus den Untersuchungen von Liebermeister wissen, nicht selten Tuberkelbacillen in Blut kreisen. Der Placentartuberkel wäre nun ebenfalls wie die erwähnten Herde in Milz, Leber und Nieren ein indifferenter pathologischer Befund, eine nicht selten, aber schwierig zu diagnosticirende Veränderung, wem er nicht an einer so exponirten Stelle, an der Grenze zwischen Mutter und Kind, gelegen wäre. Es ist ja zuerst von Gärtner und dann öfter noch und zuletzt von Sitzenfrey und Rietschel ${ }^{1}$ ) wieder darauf hingewiesen worden, dass bei der Lösung der Placenta und Eröffnung der intervillösen Räume die Placentartuberkel einreissen, die Bacillen in die Blutbahn und mit den letzten Uteruscontractionen in den Fötus geschwemmt werden können. Es besteht dieše Möglichkeit natürlich nur für die intervillösen Tuberkel, aber gerade diese Form ist, wie ja aus allen Untersuchungen hervorgeht, die häufigste, hingegen glaube ich nicht, dass aus den in der Decidua gelegenen bacillenhaltigen Rundzellenherden Bacillen leicht in den Fötus gelangen können, es sei denn, dass diese Herde ein Gefäss arrodirt haben.

Dafür, dass Tuberkelbacillen von der Mutter auf den Fötus thatsächlich übergegangen wären, bot sich bei den von mir angestellten Untersuchungen kein Anhalt. Es sind zwar alle Kirder innerhalb kurzer Zeit nach der Geburt gestorben. Tuberculöse Veränderungen aber liessen sich makroskopisch nirgends nachweisen. Ob ein Uebergang von Toxinen stattgefunden hat und so der Tod dieser Kinder - abgesehen von dem Umstand, dass sie meist Frühgeburten waren - erklärt wird, wage ich nicht zu

1) Jahrbuch für Kinderbeilkunde. Bd. 70. Heft 1. 
entscheiden. Es wäre aber meines Erachtens falsch, wollte man daraus den Schluss ziehen, dass der Placentartuberculose keine Rolle bei der Vererbung der Tuberculose zukäme. Die hier beschriebenen Untersuchungen dienten nicht direct zur Beantwortung dieser Frage, es wurden daher auch keine mikroskopischen Untersuchungen an den Fötus angestellt. Es mehren sich aber gerade auch in letzter Zeit Beobachtungen, die eine thatsächliche Uebertragung der mütterlichen Tuberculose auf das Kind durch die Placenta bestätigen oder zum Mindesten sehr wahrscheinlich machen. Ausser den älteren Fällen von Schmorl und BirchHirschfeld erwähne ich vor Allem Sitzenfrey und Rietschel. Es sind dies einmal genaue anatomische und bakteriologische Untersuchungen des Fötus und andererseits einwandfrei durchgeführte klinische Beobachtungen. Hier werden auch vor Allem künftige Untersuchungen über die Frage der congenitalen Entstehung der Tuberculose einzusetzen haben und wenn einmal eben so viele Föten tuberculöser Mütter eben so eingehend wie deren Placenten untersucht worden sind, so werden sich manche Klärungen in diesen wichtigen Fragen erzielen lassen. 